\title{
A universal formula for network functions
}

\section{Skelboe, Stig}

Published in:

IEEE Transactions on Circuits and Systems

Publication date:

1975

Document Version

Publisher's PDF, also known as Version of record

Link back to DTU Orbit

Citation (APA):

Skelboe, S. (1975). A universal formula for network functions. IEEE Transactions on Circuits and Systems, 22(1), 58-60.

\section{General rights}

Copyright and moral rights for the publications made accessible in the public portal are retained by the authors and/or other copyright owners and it is a condition of accessing publications that users recognise and abide by the legal requirements associated with these rights.

- Users may download and print one copy of any publication from the public portal for the purpose of private study or research.

- You may not further distribute the material or use it for any profit-making activity or commercial gain

- You may freely distribute the URL identifying the publication in the public portal

If you believe that this document breaches copyright please contact us providing details, and we will remove access to the work immediately and investigate your claim 


\section{A Universal Formula for Network Functions STIG SKELBOE}

\begin{abstract}
A linear electrical network can be described in a convenient way by means of the node equations. This letter presents a universal formula which expresses any network function as the quotient of two determinants. The determinants belong to matrices derived from the indefinite nodal admittance matrix $\underline{\underline{Y}}$.
\end{abstract}

\section{INTRODUCTION}

The main objective of an analysis of a linear circuit is to obtain some information on a network function. Analysis programs, such as ECAP [1], compute the modulus and argument of a network function frequency by frequency. Other programs, such as CORNAP [2], [3] and ANP3 [4], compute the poles, zeros, and gain factor of a network function. This approach is advantageous when a large number of points are wanted on a frequency response curve. The placing of the poles and zeros is also of great interest in many problems; for example, in filter design.

A characteristic for programs like CORNAP and ANP3 is that the poles and zeros are computed directly by means of eigenvalue techniques. The addition and subtraction of polynomials and computation of roots is thus avoided, and it should be avoided as it can give rise to serious numerical instability [5].

CORNAP and ANP3 are representative of the state-variable analysis formulation and the nodal analysis formulation, respectively. The state equations are difficult to establish but straightforward to solve, and conversely, the nodal equations are easily formulated but more difficult to solve.

A circuit containing no inductors has an indefinite admittance matrix [6] of the form $Y=G+s C$, where $s$ is the complex frequency (inductors may be included by gyrator-capacitor simulation). Any network function $H(s)$ can be computed from the first and second cofactors of $Y$. The two-sets-of-eigenvalues technique [7] requires, however, that $H(s)$ can be expressed as the ratio of the determinants of just two matrices which are linear in $s$. This problem has been partly solved in [8] which gives formulas for the network functions $Z_{21}, V_{21}$, and $Z_{11}$ $\left(=1 / Y_{11}\right)$. The results from [8] have been utilized in the analysis program NAPPE [9], where it is possible to retain some of the parameters as symbols.

In the following, we describe a new formula, which is used in ANP3 for computation of network functions, including $I_{21}$ and $Y_{21}$, by means of the two-sets-of-eigenvalues technique. The matrices, the determinants of which are computed, are, in general, different from the corresponding matrices used in [8].

\section{II: Definitions and Notation}

A network function is defined by a source/detector configuration. Fig. 1 shows the network connected to a voltage or current source $S$ at nodes $p$ and $q$, and connected to a voltage or current detector $D$ at nodes $r$ and $s$. The signs and arrows define the voltage polarities and current directions assumed in the formula.

In the following, the notation that will be used is

\begin{tabular}{|c|c|}
\hline$\underline{Y}_{x y z}^{a b c \cdots}$ & $\begin{array}{l}\text { matrix } \underline{Y} \text { with rows } a, b, c, \cdots \text { and columns } x, y, z, \cdots \\
\text { deleted }\end{array}$ \\
\hline$=\left(R_{\mathrm{a}} \rightarrow R_{\mathrm{b}}\right)$ & $\begin{array}{l}\text { element at position }(r, s) \text { in } \underline{\underline{Y}} \\
\text { matrix } \underline{Y} \text { after the row operation row } b:=\text { row }\end{array}$ \\
\hline
\end{tabular}

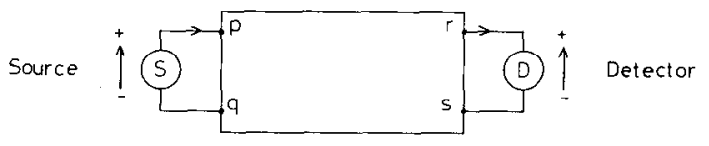

Fig. 1.

$\underset{\underline{Y}}{Y}\left(C_{a} \rightarrow C_{b}\right)$ matrix $\underline{\underline{Y}}$ after the analogous column operation $\begin{array}{ll}K_{r s} & \text { the determinant of } \underline{\underline{Y}} \\ & \text { the cofactor of element } y_{r s} \text { in } \underline{\underline{Y}} \text {. }\end{array}$

No renumbering is performed when rows or columns are deleted.

The operations previously given may appear in different combinations, e.g., $Y\left(R_{p} \rightarrow R_{q}, C_{p} \rightarrow C_{q}\right)_{s r}^{s p}$ : perform the row operation $R_{p} \rightarrow R_{q}$, the column operation $C_{p} \rightarrow C_{q}$, delete rows $s$ and $p$, and delete columns $s$ and $r$. Compute the determinant.

\section{The General Formula}

\section{Denominator}

$Y$ (the indefinite admittance matrix) is modified as follows.

1) Perform the row operation $R_{p} \rightarrow R_{q}$ and the column operation $C_{p} \rightarrow C_{q}$.

2) Delete row $s$ and column $s$.

3) For a voltage source, delete row $p$ and column $p$. For a current detector, delete row $r$ and column $r$.

The determinant of the final matrix is the denominator of the network function.

\section{Numerator}

$Y$ is modified as follows.

1) The same as 1) for the denominator.

2) The same as 2) for the denominator.

3) Delete row $p$ and column $r$.

The determinant of the final matrix is the numerator of the network function.

\section{Sign}

The sign is determined by the numerator. Let $p^{\prime}$ and $r^{\prime}$ be the actual row number and column number of row $p$ and column $r$ after execution of (2). The sign of the network function is then the sign of

$$
(-1)^{\left(p^{\prime}+r^{\prime}\right)}
$$

The formula is valid also in the following degenerate cases.

1) node $q=$ node $s$ : three-terminal two-port function;

2) node $q=$ node $s$ where this node is isolated: one-port admittance; and

3) node $p=$ node $r$ and node $q=$ node $s$ : one-port impedance. For some source-detector configurations, the universal formula leads to superflous row and column operations. These operations can be easily avoided, however, in a computer implementation.

\section{Derivation of the Formula}

In the derivation, we need the well-known properties of the indefinite admittance matrix and the following lemma which is valid for a general square matrix.

\section{Lemma}

For the $n \times n$ matrix $\underline{\underline{Y}}$ where $q \neq s$ and $q \neq r$, we have

$$
\text { a) } K\left(C_{q} \rightarrow C_{s}\right)_{q q}=K_{q q}-K_{q s}
$$

and analogously

$$
\text { b) } K\left(R_{q} \rightarrow R_{r}\right)_{q q}=K_{q q}-K_{r q} \text {. }
$$




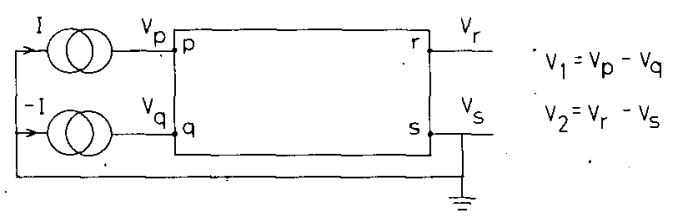

Fig. 2.

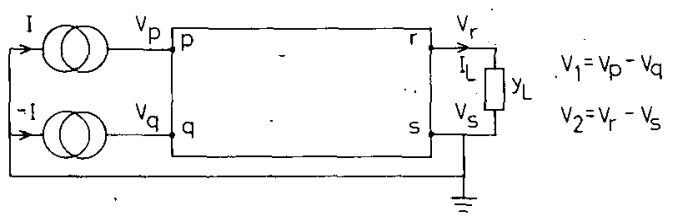

Fig. 3.

Proof: As the relations are quite analogous, only the first one is proved.

a) Assume $q<s$. $K\left(C_{q} \rightarrow C_{s}\right)_{q q}$ and $K_{q q}$ are computed by decomposition after column $s$, and $K_{q s}$ is computed by decomposition after column $q$, as seen by the equations

$$
\begin{aligned}
K\left(C_{q} \rightarrow C_{s}\right)_{q q}= & \sum_{i=1}^{q-1}(-1)^{(s-1+i)} Y_{q s}^{q i}\left(y_{i q}+y_{i s}\right) \\
& +\sum_{i=q+1}^{n}(-1)^{(s-1+i-1)} Y_{q s}^{q i}\left(y_{i q}+y_{i s}\right) \\
K_{q q}-K_{q s}= & \left(\sum_{i=1}^{q-1}(-1)^{(s-1+i)} Y_{q s}^{q i} y_{i s}\right. \\
& \left.+\sum_{i=q+1}^{n}(-1)^{(s-1+i-1)} Y_{q s}^{q i} y_{i s}\right) \\
& -(-1)^{(q+s)}\left(\sum_{i=1}^{q-1}(-1)^{(i+q)} Y_{s q}^{q i} y_{i q}\right. \\
& \left.+\sum_{i=q+1}^{n}(-1)^{(i+q-1)} Y_{s q}^{q i} y_{i q}\right) .
\end{aligned}
$$

As

$$
\begin{gathered}
Y_{q s}^{q l}=\dot{Y}_{s q}^{q l} \\
-(-1)^{(q+s)}(-1)^{(l+q)}=-(-1)^{(s+i)}=(-1)^{(s-1+i)}
\end{gathered}
$$

and

$$
-(-1)^{(q+s)}(-1)^{(i+q-1)}=-(-1)^{(i+s-1)}=(-1)^{(s-1+t-1)}
$$

we have $K\left(C_{q} \rightarrow C_{s}\right)_{q q}=K_{q q}-K_{q s}$.

b) Assume $q>s$. In this case, the cofactors of the decomposition change signs, and the proof is unchanged.

\section{Transfer Function $Z_{21}$}

In Fig. 2, node $p, q, r$, and $s$ are different (two-port function), or node $q=$ node $s$ (three-terminal two-port function). Note

$$
\underline{\underline{Y}} s \cdot \underline{V}=(0, \cdots, I, \cdots,-I, \cdots, 0)^{T} .
$$

Perform the row operation $R_{p} \rightarrow R_{q}$ such that

$$
\underline{\underline{Y}}\left(R_{p} \rightarrow R_{q}\right)_{s}^{s} \underline{V}=(0, \cdots, \cdots, 0, \cdots, 0)^{T} .
$$

Cramer's rule gives

$$
\begin{aligned}
Z_{21}=\frac{V_{2}}{I} & =(-1)^{\left(p^{\prime}+r^{\prime}\right)} \frac{Y\left(R_{p} \rightarrow R_{q}\right)_{s r}^{s p}}{Y\left(R_{p} \rightarrow R_{q}\right)_{s}^{s}} \\
& =(-1)^{\left(p^{\prime}+r^{\prime}\right)} \frac{Y\left(R_{p} \rightarrow R_{q}, C_{p} \rightarrow C_{q}\right)_{s r}^{s p}}{Y\left(R_{p} \rightarrow R_{q}, C_{p} \rightarrow C_{q}\right)_{s}^{s}}
\end{aligned}
$$

where $p^{\prime}$ and $r^{\prime}$ are row number and column number of row $p$ and column $r$ after removal of row $s$ and column $s$.

Transfer Function $V_{21}$

$$
V_{21}=\frac{V_{2}}{V_{1}}=\frac{\frac{V_{2}}{I}}{\frac{I}{I}}=\frac{Z_{21}}{Z_{0}} \quad \text { (see Fig. 2). }
$$

Cramer's rule gives

$$
Z_{0}=\frac{\dot{K}_{p p}-K_{p q}}{Y\left(R_{p} \rightarrow R_{q}\right)_{s}^{s}}
$$

where $K$ is a cofactor of the matrix $\underline{\underline{Y}}\left(R_{p} \rightarrow R_{q}\right)_{s}^{s}$. The lemma gives

$$
\begin{aligned}
Z_{0} & =\frac{K\left(C_{p} \rightarrow C_{q}\right)_{p p}}{Y\left(R_{p} \rightarrow R_{q}\right)_{s}^{s}}=\frac{Y\left(C_{p} \rightarrow C_{q}, R_{p} \rightarrow R_{q}\right)_{s p}^{s p}}{Y\left(R_{p} \rightarrow R_{q}\right)_{s}^{s}} \\
V_{21} & =(-1)^{\left(p^{\prime}+r^{\prime}\right)} \frac{Y\left(R_{p} \rightarrow R_{q}\right)_{s r}^{s p}}{Y\left(R_{p} \rightarrow R_{q}, C_{p} \rightarrow C_{q}\right)_{s p}^{s p}} \\
& =(-1)^{\left(p^{+}+r^{\prime}\right)} \frac{Y\left(R_{p} \rightarrow R_{q}, C_{p} \rightarrow C_{q}\right)_{s p}^{s p}}{Y\left(R_{p} \rightarrow R_{q}, C_{p} \rightarrow C_{q}\right)_{s p}^{s p}}
\end{aligned}
$$

Transfer Function $I_{21}$

Add $y_{L}$ to the original circuit, as seen in Fig. 3, $Y_{L}$ modifies $Y$ in four places, as seen by

$$
\begin{gathered}
\underline{Y}^{\prime}=\left[\begin{array}{cc}
y_{r r}+y_{L} & y_{r s}-y_{L} \\
y_{s r}-y_{L} & y_{s s}+y_{L}
\end{array}\right] \\
Z_{21}^{\prime}=\frac{V_{2}}{I}=(-1)^{\left(p^{\prime}+r^{\prime}\right)} \frac{Y\left(R_{p} \rightarrow R_{q}\right)_{s r}^{s p}}{Y\left(R_{p} \rightarrow R_{q}\right)_{s}^{s}+y_{L} Y\left(R_{p} \rightarrow R_{q}\right)_{s r}^{s r}} \\
I_{21}^{\prime}=\frac{I_{L}}{I}=\frac{V_{2}}{I} y_{L}=Z_{21}^{\prime} y_{L} \\
I_{21}=\lim _{y_{L} \rightarrow \infty} I_{21}^{\prime}=(-1)^{\left(p^{\prime}+r^{\prime}\right)} \frac{Y\left(R_{p} \rightarrow R_{q}\right)_{s r}^{s p}}{Y\left(R_{p} \rightarrow R_{q}\right)_{s r}^{s r}} \\
=(-1)^{\left(p^{\prime}+r^{\prime}\right)} \frac{Y\left(R_{p} \rightarrow R_{q}, C_{p} \rightarrow C_{q}\right)_{s r}^{s p}}{Y\left(R_{p} \rightarrow R_{q}, C_{p} \rightarrow C_{q}\right)_{s r}^{s r}}
\end{gathered}
$$

Transfer Function $Y_{21}$

See Fig. 3 and note

$$
Y_{21}=\lim _{y_{L} \rightarrow \infty} \frac{I_{L}}{V_{1}}=\lim _{y_{L} \rightarrow \infty} \frac{\frac{I_{L}}{I}}{\frac{V_{1}}{I}}=\frac{I_{21}}{Z_{s}}
$$




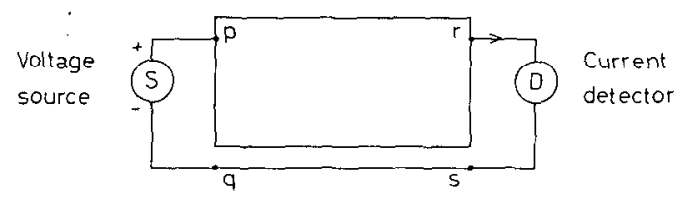

Fig. 4.

where

$$
Z_{s}=\frac{Y\left(R_{p} \rightarrow R_{q}, C_{p} \rightarrow C_{q}\right)_{s p r}^{s p r}}{Y\left(R_{p} \rightarrow R_{q}\right)_{s r}^{s r}} .
$$

The removal of row and column $r$ means that node $r$ is grounded and thus connected to node $s$.

$$
Y_{21}=\frac{I_{21}}{Z_{s}}=(-1)^{\left(p^{\prime}+r^{\prime}\right)} \frac{Y\left(R_{p} \rightarrow R_{q}, C_{p} \rightarrow C_{q}\right)_{s r}^{s p}}{Y\left(R_{p} \rightarrow R_{q}, C_{p} \rightarrow C_{q}\right)_{s p r}^{s p r}} .
$$

One-Port Impedance $Z_{11}$

Cramer's rule gives immediately

$$
Z_{11}=\frac{V_{1}}{I}=\frac{Y_{q p}^{q p}}{Y_{q}^{q}} \quad \text { (see Fig. 2). }
$$

This expression is equal to (1) when node $p=$ node $r$ and node $q=$ node $s$.

One-Port Admittance $Y_{11}$

Note

$$
Y_{11}=\frac{1}{Z_{11}}=\frac{Y_{r}^{r}}{Y_{r p}^{r p}} .
$$

Compute $Y_{21}$, according to (4), as the transfer function from the nodes $p$ and $q$ to the nodes $r$ and $s$, as seen by

$$
Y_{21}=(-1)^{\left(p^{\prime}+r^{\prime}\right)} \frac{Y^{\prime}\left(R_{p} \rightarrow R_{q}, C_{p} \rightarrow C_{q}\right)_{s r}^{s p}}{Y^{\prime}\left(R_{p} \rightarrow R_{q}, C_{p} \rightarrow C_{q}\right)_{s p r}^{s p r}} \quad \text { (see Fig. 4). }
$$

$\underline{\underline{Y}}^{\prime}$ is derived from $\underline{\underline{Y}}$ by adding a zero row and a zero column corresponding to node $q$ (= node $s$ ). That is, $\underline{\underline{Y}}_{s}^{\prime s}=\underline{\underline{Y}}$, and furthermore, $(-1)^{p^{\prime}+r^{\prime}} Y_{s r}^{\prime s p}=Y_{r}^{r}$ because all cofactors of $Y$ are equal. (This is a well-known property of the indefinite nodal admittance matrix.) Analogously, we have $Y_{s p r}^{\prime s p r}=Y_{r p}^{r p}$, and thus $Y_{11}=Y_{21}$ in the case of Fig. 4. This completes the proof of the universal formula stated in Section III.

\section{Conclusion}

The formula has lead to a very efficient organization of the two-sets-of-eigenvalues circuit analysis program ANP3 developed at the Technical University of Denmark.

\section{ACKNOWLEDGMENT}

This work is based on a less unified formula proposed by E. V. Sørensen during the development of the ANP3 program and proven by B. Guldbrandsen. Professor Sørensen's interest in this extension of his work is gratefully acknowledged.

\section{REFERENCES}

[1] "1620 electronic circuit analysis program [ECAP] [1620-EE-02X] user's manual," IBM Appl. Prog. File H20-0170-1, 1965.

[2] C. Pottle, "State-space techniques for general active network analysis," in Systems Analysis by Digital Computer, F. F. Kuo and J. F. Kaiser, Eds. New York: Wiley, 1966.

[3] L 'A 'textbook' computerized state-space network analysis algorithm," IEEE Trans. Circuit Theory, vol. CT-16, pp. 566-568, Nov. 1969.

[4] R. Molich-Pedersen, "User's manual for the ANP3 circuit analysis program," Lab. Circuit Theory, Tech. Univ. Denmark, Lyngby, Mar.
[5] J. H. Wilkinson, "Rounding errors in algebraic processes," in Notes on Appl. Science No. 32, Her Majesty's Stationery Office, London, 1963.

[6] G. E. Sharpe and B. Spain, "On the solution of networks by means of the equicofactor matrix," IRE Trans. Circuit Theory, vol. CT-7, pp. 230-239, Sept. 1960

[7] I. W. Sandberg and H. C. So, "A two-sets-of-eigenvalues approach to the computer analysis of linear systems," IEEE Trans. Circuit Theory, vol. CT-16, pp. 509-517, Nov. 1969.

[8] G. E. Alderson, "Semi-numerical analysis of large networks," Ph.D. dissertation, Dept. Elec. Eng., Purdue University, Lafayette, Ind., Aug. 1971.

[9] G. E. Alderson and P. M. Lin, "Computer generation of symbolic network functions--a new theory and implementation," IEEE Trans. Circuit Theory, vol. CT-20, pp. 48-56, Jan. 1973.

\section{Tree Graphs and Tree Numbers}

\section{S. KRISHNAMOORTHY AND NARSINGH DEO}

Abstract-The concept of the tree graph of a given connected graph was first introduced and studied by Cummins [2]. Further properties of tree graphs were explored in [1], [6]-[10].

In this correspondence, some additional properties of tree graphs are brought out. A related concept of tree numbers is introduced and explored.

\section{INTRODUCTION}

We shall consider only graphs that are nonnull, finite, undirected, connected, and simple (i.e., graphs without self loops and parallel edges), because what follows makes sense only for such graphs. Let $\dot{G}=(V, E)$ denote a finite, undirected, connected, simple graph with vertex set $V$ and edge set $E$. The tree graph $T(G)=\left(V^{1}, E^{1}\right)$ of $G$ is defined as follows. There is a one-to-one correspondence between the spanning trees of $G$ and the vertices of $T(G)$ such that two vertices in graph $T(G)$ are adjacent, if and only if the corresponding spanning trees in $\boldsymbol{G}$ are at a distance of one. ${ }^{1}$ The properties of tree graph were studied by Kamae [6], Kishi and Kajitani [7]-[9], Amoia and Cottafava [1], and Malik [10]. The motivation behind these studies has been the relevance of tree graph to generation of all spanning trees of $G$ and to central trees of $G$ [3], [4].

It has been shown [2] that for any given connected graph $G$, there exists a tree graph $T(G)$, which is also connected. Furthermore, $T(G)$ always has at least one Hamiltonian circuit. A center of $T(G)$ corresponds to a central trce.

Using Harary's [5] terminology, we denote the complete graph of $n$ vertices by $K_{n}$ and the circuit of $n$ vertices (i.e., the $n$-gon) by $C_{n}$. Let the usual symbol $\simeq$ denote the isomorphism between two graphs. Then we obtain the following results.

\section{Properties of Tree Graphs}

\section{Lemma 1}

The tree graph $T(G)$ of a graph $G$ is isomorphic to $G$, if and only if $G$ is $K_{3}$.

Proof: If $G$ is $K_{3}$, it clearly has three spanning trees, each at a unit distance from the other two, and therefore, $T\left(K_{3}\right) \simeq K_{3}$. To prove the "only if" part let. $G$ be a graph such that $T(G) \simeq G$, and let $n$ be the number of vertices in $G$ as well as in $T(G)$. Since

Manuscript received October 12, 1973.

The authors are with the Department of Electrical Engineering and Computer Centre, Indian Institute of Technology, Kanpur-16, U.P., India.

1 It is well known that the distance between two spanning trees $d\left(t_{i}, t_{j}\right)$ is defined as the number of edges present in one spanning tree but not in the other. In other words,

$$
d\left(t_{i}, t_{j}\right)=1 / 2 \quad\left|t_{i} \oplus t_{j}\right|
$$

where $\oplus$ denotes the symmetric difference of sets, and | | denotes the cardinality. That this distance among spanning trees of a given graph satisfies the usual metric properties can be easily seen. 